IAU Colloquium 164: Radio Emission from Galactic and Extragalactic Compact Sources

ASP Conference Series, Vol. 144, 1998

J. A. Zensus, G. B. Taylor, \& J. M. Wrobel (eds.)

\title{
Structural Variations in the Quasar 3C 454.3
}

\author{
I. I. K. Pauliny-Toth
}

Max-Planck-Institut für Radioastronomie, Bonn, Germany

Abstract. Imaging of the OVV quasar 3C $454.3(z=0.859)$ shows that a new component was ejected from the core at epoch $1981 \pm 1$, close to the time of a major radio outburst. The component moved along a curved path with a mean apparent velocity of $\sim 17 c\left(H_{o}=100 \cdot h\right.$ $\left.\mathrm{km} \mathrm{s}^{-1} \mathrm{Mpc}^{-1}\right)$. The motion, however, showed variations: from $\sim 11 c(1984-1985)$, to $\sim 22 c$ (1985-1988.5), then to $\sim 14 c(1988.5-1991.9)$.

At epoch $\sim 1980$, a strong radio outburst began in 3C 454.3. Subsequent global VLBI observations at $\lambda 2.8 \mathrm{~cm}$ revealed a complex structure, composed of three stationary components and two weak features which appeared to recede from the core along P.A. $-95^{\circ}$ with velocities of $\sim 9 c$ and $\sim 5 c$ (Pauliny-Toth et al. 1987, PT87). Global VLBI observations were also made at $\lambda 6 \mathrm{~cm}$, at $2-3 \mathrm{yr}$ intervals. Images from these are shown in Fig. 1.

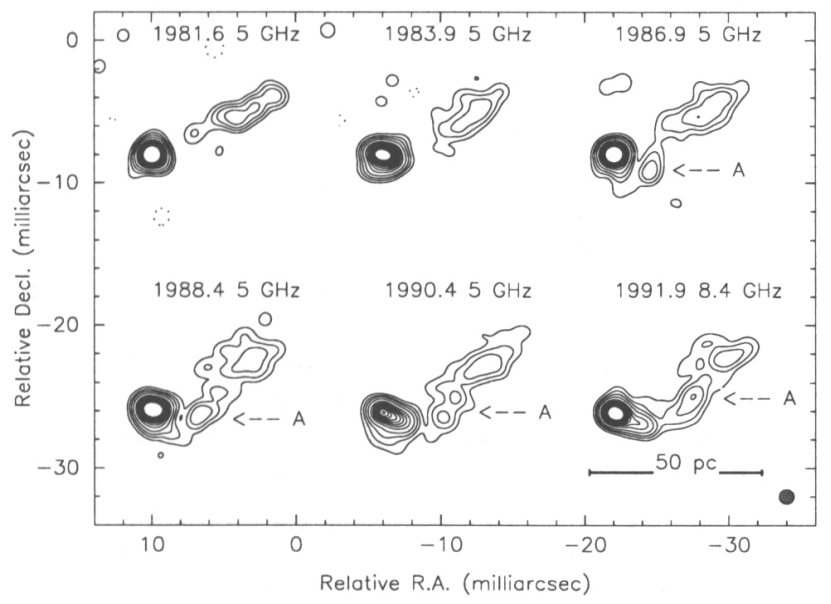

Figure 1. Images of $3 \mathrm{C} 454.3$ derived from global VLBI observations at $5 \mathrm{GHz}$ (first 5 epochs) or $8.4 \mathrm{GHz}$ (last epoch). The restoring beam is a circular gaussian with FWHM 1 mas. Contour levels are $(-1,1,2.5,5,10,20 \ldots 100) \cdot 35 \mathrm{mJy} /$ beam area, except for the last epoch, where the scaling factor is $24 \mathrm{mJy} /$ beam area.

We associate the feature(s) labeled " $A$ " with the weak, moving components detected at $\lambda 2.8 \mathrm{~cm}$. "A" moves away from the core along a curved path. Its distance from the core, measured along this path, is shown in Fig. 2.

The results are as follows:

1) Component A moves along a curved path towards the "mas jet" which was already present at epoch 1981.6.

2) Its velocity along this path changes from $\sim 11 c(1984-1985)$, to $\sim 22 c(1985-$ $1988.5)$ and then to $\sim 14 c(1988.5-1991.9)$. The mean velocity over the whole range of epochs is $0.68 \pm 0.02 \mathrm{mas} / \mathrm{yr}$, or $(17.2 \pm 0.6) c$. These velocities are higher than the value of $\sim 5 c$, for superluminal sources in general (e.g., Porcas 1987). 


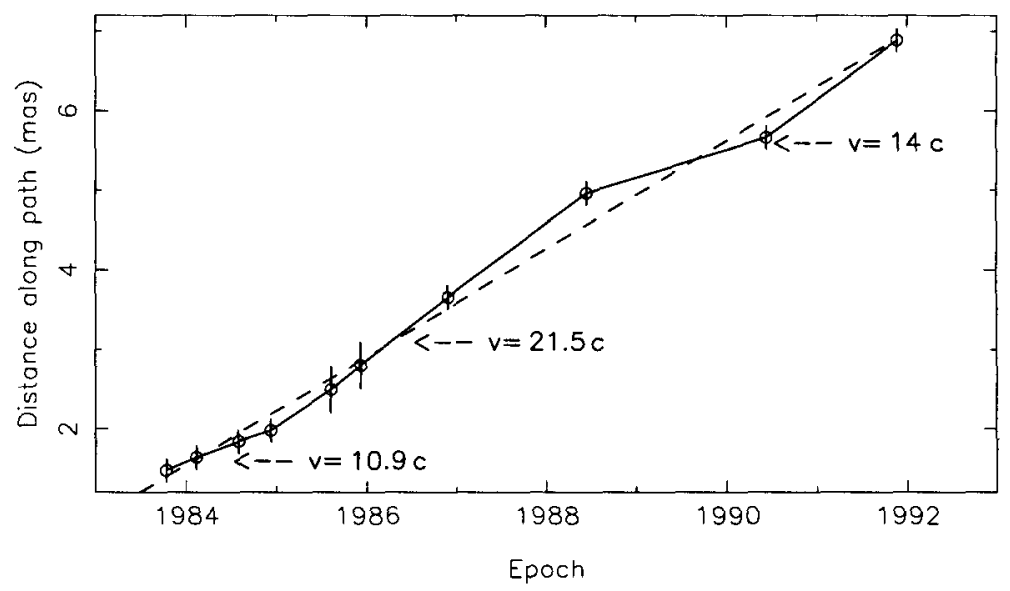

Figure 2. The distance traveled by component A, along its curved path, vs epoch. The velocities indicated are for epochs 1983-1985, 1985-1988.5 and 1988.5-1991.9.

3) The epoch of ejection, using a straight line fit to all the epochs is $1981.7 \pm 0.8$; for only the first 4 epochs, it is $1980.3 \pm 1.0$. The latter value corresponds closely to the beginning of the outburst.

4) The distance of the mas jet from the core at 1981.6 is 8.2 mas. The last major outburst in 3C 454.3 before 1981.6 began at $\sim 1966.0$ (Kellermann \& PaulinyToth 1981). If this jet originated in that outburst, the velocity would have been $\sim 0.53 \mathrm{mas} / \mathrm{yr}$ or $\sim 13 c$, a value close to those obtained for component $\mathrm{A}$. It thus seems likely that the mas jet originated in the 1966 outburst.

5) The stationary features in the core region reported in PT87 may be simply explained in terms of motion of the jet along a sinusoidal path. A simple, 2-D model is of such a path, with amplitude $\sim 0.15 \mathrm{pc}$, period $\sim 40 \mathrm{pc}$ and axis oriented at $3^{\circ}$ to the line of sight. The jet has a Lorent $z$ factor $\gamma \sim 20$ and the stationary features represent the points where the jet direction is along the line of sight. This naive model reproduces the observed spacings of the features. At some distance from the core (1-1.5 mas) the path presumably changes and a moving shock (e.g., component A) develops.

Acknowledgments. This work was carried out in collaboration with $R$. W. Porcas (MPIfR), J. A. Zensus (NRAO) and G. D. Nicolson (NITR, Johannesburg). The National Radio Astronomy Observatory is a facility of the National Science Foundation, operated under a cooperative agreement by Associated Universities, Inc.

\section{References}

Kellermann, K. I., \& Pauliny-Toth, I. I. K. 1981. ARA GA, 19, 373-410.

Pauliny-Toth, I. I. K., et al. 1987. Nature, 328, 778-782.

Porcas, R. W. 1987. in Superluminal Radio Sources, eds. J. A. Zensus \& T. J. Pearson (Cambridge: Cambridge Univ. Press), 12-25. 